\title{
Perfil profesional de los fonoaudiólogos colombianos que trabajan con adultos mayores
}

\section{Professional profile of Colombian speech and language pathologists that work with elderly persons}

\begin{abstract}
RESUMEN
El objetivo del estudio fue caracterizar el perfil profesional de fonoaudiólogos que trabajan con adultos mayores en Colombia. Metodología: La investigación fue de tipo descriptivo; los participantes fueron 30 fonoaudiólogos quienes se encargaron de responder una encuesta. La encuesta contenía ítems relacionados con el perfil sociodemográfico, el perfil profesional, el quehacer fonoaudiológico y las percepciones sobre la población adulta mayor. Resultados: El abordaje fonoaudiológico con esta población se caracteriza por estar centrado en el escenario salud, en su mayoría el rol es el asistencial, específicamente en los procesos de evaluación e intervención en las áreas de deglución, lenguaje y comunicación Conclusiones: Los resultados permitieron caracterizar el perfil profesional y el quehacer del fonoaudiólogo con la población adulta mayor. También evidencian la importancia de continuar el trabajo con este grupo etario en función de sus habilidades y bienestar comunicativo, y de allí la necesidad de plantear estudios que pueden servir para comprender la comunicación en población adulta con y sin desórdenes.
\end{abstract}

Palabras clave: Fonoaudiólogo, perfil profesional, adulto mayor, alcance de la práctica.

\begin{abstract}
The aim of the study was to characterize the professional profile of speech therapists who work with elderly adults in Colombia. Methodology: The study was descriptive; participants were 30 speech and language pathologists who answered a survey including items related with sociodemographic profiles, professional profiles, speech and language pathology tasks, and perceptions about the elder adults. Results: The approach to speech and language pathology practice with this population is focused on the health setting, is mainly centered on assisting patients on areas such as swallowing, language, and communication. Conclusions: The results allowed characterizing the professional profile and the speech and language pathologist's practice with the elderly adult population. They also showed the importance of working with elderly persons based on their abilities and their communicative well-being. Also, it is necessary to conduct further studies to understand communication among elderly adults with and without disorders.
\end{abstract}

Keywords: speech and language pathologist, professional profile, elderly people, scope of practice. 


\section{Introducción}

El envejecimiento desde una visión sociocultural es construido desde diferentes significados según la época, la cultura y la sociedad. Para Dulcey-Ruiz, Arrubla, \& Sanabria (2013), es un proceso progresivo, intrínseco y universal que con el tiempo ocurre en todo ser vivo como consecuencia de la interacción de la genética del individuo y su medio ambiente: "no solo tiene que ver con procesos biológicos, sino que es moldeado por normas o factores culturales $y$ sociales" (p. 16).

El adulto participa y se desenvuelve en contextos que va construyendo a lo largo de su vida, los cuales benefician su participación y le ayudan a construir un conjunto de saberes, habilidades y destrezas que le van a permitir comunicarse e integrarse efectivamente con otros seres. Estos contextos (familiares, profesionales, recreativos, sociales y laborales) aportan a su desarrollo social y fortalecen la competencia comunicativa, la cual le permite ejercer los roles propios de su edad (Rodríguez, 2009 , p. 1). Durante la vejez ocurren cambios anatómicos y funcionales en el sistema nervioso, en los órganos de los sentidos, en las funciones cognitivas, en la afectividad (pérdidas, motivaciones), en la personalidad y en el rol social, los que impactan en la vida del individuo (Millán, 2006).

Según Petersen (2004) en el adulto mayor se dan cambios socioculturales y físico-orgánicos. Los primeros están relacionados con las transformaciones de la rutina diaria, lo que los obliga a quedarse solos en sus hogares lo cual provoca cambios en su desempeño social y productivo. Los segundos, los físico-orgánicos están asociados a los cambios en las funciones sensoriales, cognoscitivas y motoras. De allí la importancia de realizar un trabajo multidisciplinario en el que se unan diversas perspectivas y abordajes a partir del cual se brinda una atención integral a la población mayor y se influye de manera positiva en su salud y en su calidad de vida (Berrío, 2012).

Al revisar la literatura se observa que a investigación desarrollada en torno al abordaje fonoaudiológico en la población adulta mayor tiende a centrarse en la descripción de los factores neurológicos $\mathrm{y}$ de envejecimiento cognitivo que afectan a los sujetos y que causan un problema de comunicación. Mientras que en menor proporción, se encuentran estudios orientados a la identificación y a la descripción de las funciones de intervención y evaluación-diagnóstica desde una perspectiva de deficiencia comunicativa y no desde un proceso normal de envejecimiento.

En relación con las investigaciones que se han centrado en los profesionales que trabajan con población adulta mayor este es escaso. Montoya \& Rodríguez (2018) reconocen el papel que desempeña el fonoaudiólogo por ser el profesional de la comunicación humana y sus desórdenes y es quien busca el bienestar comunicativo más allá de la deficiencia que pueda llegar a presentar. En este estudio, las autoras concluyeron que era necesario generar más investigaciones que permitan describir la importancia de este profesional en la prestación de servicios dirigidos a este grupo etario. Lo anterior permitiría generar cambios en torno a las percepciones sobre las habilidades comunicativas, la participación social, la calidad de vida y el bienestar comunicativo de esta población con y $\sin$ 
envejecimiento patológico. De igual manera, enfatizan la importancia de favorecer cambios que permitan proponer políticas públicas y programas que busquen el bienestar comunicativo y la calidad de vida de esta población y de sus contextos más cercanos. En este contexto las autoras asumen el bienestar comunicativo como el "estado de óptima capacidad que podría alcanzar quienquiera en cualquier etapa del ciclo de la vida" (Cuervo, 1998, p. 35).

La falta de investigaciones centradas en el desempeño fonoaudiológico con la población adulta, el incremento demográfico, el establecimiento y difusión de nuevas políticas, y los cambios que se dan en esta etapa de vida relacionados con la comunicación y con la participación social, motivaron la formulación de un estudio que busca caracterizar el perfil sociodemográfico, el quehacer fonoaudiológico y las percepciones de los fonoaudiólogos sobre la población adulta mayor. A continuación se expone el estudio desarrollado.

Antes de iniciar es importante mencionar que en relación a la percepción, en este estudio se asume que:

La formulación de juicios no es un proceso lineal de estímulo y respuesta sobre un sujeto pasivo, sino que, por el contrario, están de por medio una serie de procesos en constante interacción y donde el individuo y la sociedad tienen un papel activo en la conformación de percepciones particulares a cada grupo social. En el proceso de la percepción están involucrados mecanismos vivenciales que implican tanto al ámbito consciente como al inconsciente de la psique humana (Vargas, 1994, p. 48).

\section{Metodología}

\section{Tipo de estudio}

Esta investigación tuvo un alcance descriptivo. Según Hernández, Fernández, \& Baptista (2010), los estudios descriptivos permiten "describir fenómenos, situaciones, contextos y eventos; esto es, detallar cómo son y cómo se manifiestan" (p. 80). Particularmente, en esta investigación se buscó caracterizar el perfil sociodemográfico, el perfil profesional, el quehacer fonoaudiológico y las percepciones que tienen los fonoaudiólogos de la población adulta mayor en Colombia.

\section{Participantes}

En el estudio participaron 30 fonoaudiólogos colombianos. Los criterios para la participación fueron: ser fonoaudiólogos y haber trabajado o trabajar con población adulta mayor. El $87 \%$ de los participantes (26 personas) eran mujeres y el $13 \%$ (4) hombres. La edad mínima fue de 30 y la máxima de 53 años (media $=34$ añosdesviación estándar $=$ $9,157)$. En relación con los años de experiencia con la población adulta mayor, el $57 \%$ tenía entre 1 y 5 años de trabajo con dicha población, el 23\% entre 6 y 10 años, el $23 \%$ entre 11 y 20 años, y el 7\% más de 20 años.

\section{Instrumentos}

Para recolectar información se utilizó un instrumento no estandarizado tipo encuesta diseñado por las fonoaudiólogas investigadoras. Mayntz \& et al., (1976) citados por Díaz de Rada 
(2007) definen la encuesta como la búsqueda sistemática de información en la que el investigador pregunta a los investigados sobre los datos que desea obtener, y posteriormente los reúne los analiza en forma pertinente. Gracias a ella se pueden explorar según Grasso (2006, p. 13) cuestiones basadas en la subjetividad y al mismo tiempo facilita obtener información de un número considerable de personas.

La encuesta constó de 38 preguntas divididas en dos partes. La primera parte contenía una breve introducción del contenido y los datos personales del encuestado (edad, nacionalidad, género, lugar de procedencia y lugar de trabajo). La segunda parte estaba conformada por cuatro sub-partes, a saber, el perfil sociodemográfico (4 preguntas), el perfil profesional (12 preguntas), el quehacer profesional (15 preguntas) y la percepción de los profesionales acerca de la persona mayor (7 preguntas). Cada subparte contenía preguntas abiertas, de selección múltiple y dicotómica (sí-no). La encuesta se diligenció en formato electrónico para facilitar su diligenciamiento y el envío de la información.

\section{Fases y procesamiento de datos}

La investigación se llevó a cabo en tres fases: (1) diseño y validación del instrumento, (2) recolección y tabulación de datos, y (3) análisis de la información.

La primera fase implicó la organización de un marco teórico para identificar las variables; posteriormente se seleccionó el tipo de instrumento, eligiendo el uso de la encuesta. Para la validación del instrumento se utilizó el juicio de expertos. Para Escobar \& Cuervo (2008, p. 28) el juicio de expertos es importante debido a que la trayectoria y a la experiencia de los expertos en su campo de conocimientos permite la valoración de instrumentos para ser aplicados con más confiabilidad. En el proceso de validación, participaron 5 expertos, todos ellos fonoaudiólogos, con experiencia en población adulta. Para la evaluación del instrumento por parte de los expertos se diseñó un formato electrónico que fue enviado a cada uno de los profesionales y que contaba de criterios como pertinencia, suficiencia, coherencia y cohesión. Al finalizar esta fase se realizaron los ajustes al instrumento teniendo en cuenta las calificaciones y observaciones de los jueces.

La segunda fase se orientó a la recolección de los datos. Para tal efecto, se envió un enlace virtual para acceder a la encuesta a cada uno de los fonoaudiólogos encuestados a través de un formato de Google. Los profesionales fueron contactados vía internet. Antes de diligenciar la encuesta los participantes firmaron un consentimiento informado de manera electrónica. El tiempo destinado para la recolección de los datos fue de tres meses.

En la tercera fase los datos fueron tabulados en una matriz en Excel que fue diseñada por las fonoaudiólogas investigadoras. Se realizó una estadística descriptiva para identificar las respuestas a cada uno de los ítems cerrados con el programa SPSS, y las respuestas abiertas fueron analizadas para identificar categorías de análisis.

\section{Resultados}

\section{Perfil sociodemográfico}

Los fonoaudiólogos encuestados pertenecían a diferentes localidades de Colombia: 12 personas 
eran de la capital del país (Bogotá D.C), 4 de Cali (occidente del país, capital del Departamento del Valle del Cauca), 2 de Bucaramanga (nororiente del país, capital del Departamento de Santander), 2 de Popayán (suroccidente del país, capital del Departamento del Cauca), 1 de Armero Guayabal (centro-oeste del país, municipio ubicado en el Departamento del Tolima), 1 de Cúcuta (nororiente del país, capital del Departamento de Norte de Santander),1 de Ibagué (centro-occidente del país, capital del Departamento del Tolima), 1 de Manizales (centro-occidente del país, capital del Departamento de Caldas), 1 de Planeta Rica (norte del país, municipio ubicado en el Departamento de Córdoba), 1 de Sibaté (centro del país, municipio ubicado en el Departamento de Cundinamarca), 2 de Boyacá (Departamento ubicado en el centro-este del país), 2 de Huila (Departamento ubicado al suroeste del país) y 1 de Nariño (Departamento ubicado en el extremo suroeste del país). De estos lugares el $90 \%$ corresponde a sitios urbanos y el $10 \%$ a rurales.

\section{Perfil profesional}

En relación con el centro de formación, el $40 \%$ de los profesionales era egresados de la Universidad Nacional de Colombia (ubicada en la ciudad de Bogotá D.C) seguido por la Universidad de Pamplona (Departamento de Norte de Santander) con un 14\%. Luego se encontraban los egresados de la Universidad del Rosario (0\%) de la Universidad Manuela Beltrán (10\%) y de la Corporación Universitaria Iberoamericana (10\%, Bogotá D.C.). En un menor porcentaje se encontraban la Universidad del Cauca (7\%,Departamento del Cauca), la Universidad Metropolitana (3\%, Barranquilla, Capital del Departamento del Atlántico), la Universidad Santiago de Cali (3\%, Cali, Departamento del Valle del Cauca), y la Unisucre (3\%, Departamento de Sucre).

En cuanto a la variable tiempo de egreso, el $47 \%$ de los participantes había egresado entre 1 y 5 años, el 33\% entre 11 y 20 años, el 7\% entre 6 y 10 años, y el $13 \%$ más de 20 años.

La siguiente variable era el grado educativo, en éste el $50 \%$ tenía sólo el de fonoaudiólogos, el $23 \%$ de maestría, el $20 \%$ de especialización y el $7 \%$ de doctorado. Las áreas de formación fueron: a) Especialización: Deglución y Salud Pública; y b) Maestría: Comunicación, lenguaje, habla, audición, vías aerodigestivas superiores, miofuncional, neurociencias, y educación.

En relación a la edad de las personas adultas con quienes trabajaban. El $84 \%$ de los fonoaudiólogos había trabajado con población adulta mayor entre 60 y 80 años mientras que el 16\% lo había hecho con adultos mayores de 81 años. El $86 \%$ de los fonoaudiólogos había abordado la población adulta mayor con un envejecimiento patológico mientras que el $14 \%$ lo había hecho con población con envejecimiento típico.

En cuanto a las áreas más representadas, el área de deglución apareció como la más abordada, con el $50 \%$ de los fonoaudiólogos encuestados reportando trabajar en esta área, luego se encontraban lenguaje con el $25 \%$ y comunicación con el $25 \%$. Lo anterior evidencia una tendencia a trabajar con esta población desde un modelo clínico en el que la deglución, por ejemplo, se asume como un deterioro asociado al envejecimiento el cual puede ocasionar una disfagia o una presbifagia. En el área de 
lenguaje, es importante mencionar que entre las deficiencias se encontraban los trastornos cognitivocomunicativo asociados a enfermedades neurodegenerativas, y las afasias que se dan como producto de accidentes cerebrovasculares $\mathrm{y} / \mathrm{o}$ traumas craneoencefálicos.

En cuanto a las deficiencias comunicativas, los fonoaudiólogos reportaron intervenir los siguientes aspectos: a) limitación en la interacción debido a dificultades en el inicio, mantenimiento y finalización de temas conversacionales, y b) dificultades en la organización social del discurso que afectan las interacciones en las que participan los adultos mayores.

\section{Quehacer profesional}

En la variable quehacer profesional el $66 \%$ de los fonoaudiólogos ejercía el rol asistencial, el $17 \%$ el investigativo, el $10 \%$, el administrativo, y el $7 \%$ el docente. Los fonoaudiólogos que ejercían el rol asistencial realizaban funciones de evaluación diagnóstico y de intervención/rehabilitación. Los que ejercían el rol investigativo realizaban estudios en áreas como: comunicación adulta, desórdenes comunicativos, desórdenes de vías aerodigestivas superiores, alteraciones auditivas, y desórdenes de voz. Los fonoaudiólogos que ejercían el rol administrativo eran coordinadores de proyectos de mejoramiento social, diseñaban y ejecutan programas fonoaudiológicos, y eran asesores en proyectos. Por su parte, los fonoaudiólogos que ejercían el rol docente trabajan en la educación formal. En cuanto a los escenarios, el $70 \%$ de los fonoaudiólogos trabajaba en salud específicamente en hospitales y consultorios privados, el $23 \%$ ejercía su rol en el escenario de Bienestar Social con fundaciones y en centros de día - noche, y el 7\% laboraba en establecimientos de educación. Es importante mencionar que el $56 \%$ de las instituciones en las cuales trabajaban los profesionales de la comunicación humana y sus desórdenes eran de carácter privado mientras que el $44 \%$ eran públicas; el $70 \%$ de ellas estaban ubicadas en áreas urbanas y el 30\% en áreas rurales.

En relación con las funciones, el $35 \%$ de los fonoaudiólogos ejercía la función de evaluacióndiagnóstico, el 40\% de intervención-rehabilitación, el 15\% de asesoría-consejería, el 5\% de promoción, y el $5 \%$ de prevención. En el procedimiento de evaluación-diagnóstico el profesional de la comunicación humana y sus desórdenes tenía en cuenta: la interacción comunicativa, la participación social, las deficiencias de base, las habilidades comunicativas conservadas, las habilidades comunicativas alteradas y los signos lingüísticos de los adultos mayores. Respecto a las estrategias empleadas se observó lo siguiente: En la evaluacióndiagnóstica, el $57 \%$ de los profesionales usaban instrumentos no estandarizados, el $20 \%$ de instrumentos estandarizados, y el $23 \%$ empleaba la observación. En cuanto al enfoque, el $70 \%$ de los fonoaudiólogos asumía su práctica desde un modelo individual (médico), el $15 \%$ desde un modelo social (CIF y rehabilitación), el $8 \%$ desde un modelo integral (modelo sistémico de la comunicación), y el 7\% utilizaba un modelo biopsicosocial.

En promoción, el 35\% realizaba campañas de estimulación cognitiva-comunicativa, el $17 \%$ usaba estrategias de pautas de ingesta oral segura, el $10 \%$ empleaba estrategias interactivas dirigidas a 
interlocutores, resaltando el papel de la interacción durante la ingesta oral, El 5\% realizaba capacitaciones sobre el manejo de texturas, el $5 \%$ hacía promoción de la salud auditiva, el 5\% trabajaba con salud vocal, el $5 \%$ con habilidades comunicativas, el $5 \%$ con participación social, y el $3 \%$ con sensibilización acerca de la comunicación y la participación de la persona mayor. En prevención, el $50 \%$ de los fonoaudiólogos identificaba en forma temprana los desórdenes comunicativos y el $50 \%$ se centraba en la caracterización del desorden comunicativo. Por último, para las funciones de asesoría y consejería, el $23 \%$ de los profesionales trabajaba con la familia, el $38 \%$ con otros profesionales y con la familia, y el $35 \%$ con profesionales, familiares, organizaciones y comunidades donde se desenvuelven las personas mayores.

\section{Percepciones de los profesionales en torno al adulto mayor}

En este apartado se presentan los juicios/visiones que han construido los profesionales de la comunicación humana y sus desórdenes del adulto mayor.

El 97\% valoró el trabajo con esta población pues a través de diferentes acciones trabajaban a favor de la calidad de vida y del bienestar comunicativo y esto les ayudaba a ejercer diferentes roles y a participar en diversos contextos sociales. El $40 \%$ de los encuestados se interesaba en aumentar las interacciones y la participación social. El 35\% fomentaba la calidad de vida y el bienestar comunicativo. Finalmente, el $25 \%$ orientaba su intervención a los cuidadores y/o interlocutores para brindarles estrategias que les permitan mejorar las habilidades comunicativas y así contribuir a lograr una mayor participación en las interacciones en los diferentes contextos.

Frente a las razones que los llevaron a trabajar con este grupo etario: el $40 \%$ lo hizo por oferta laboral; el $27 \%$ porque quería un cambio en cuanto al abordaje de la vejez como una etapa más del ciclo vital y no desde la deficiencia; el $23 \%$ por influencia de la formación académica recibida; y el 10\% por un interés personal.

Sin duda uno de los puntos a destacar se encuentra relacionado con la importancia que le dan los fonoaudiólogos a la descripción del adulto mayor desde una visión paciente-patología. Lo anterior hace que el modelo de abordaje sea individual, que se enfoque el abordaje a la dimensión parte personal y no se trabaje con los agentes que comparten con ellos en los diferentes contextos y que pueden aportar a la calidad de vida y al bienestar comunicativo.

Para finalizar, los resultados muestran que el concepto del abordaje del fonoaudiólogo está centrado en lo clínico, en la explicación y rehabilitación de las deficiencias que afectan a esta población, en mantener las habilidades que aún permanecen en la búsqueda de programas que permitan superar las deficiencias. De allí la necesidad de trabajar en la sensibilización, en la descripción del mundo del adulto mayor, de sus capacidades $y$ habilidades, y de los contextos en los que participa. 


\section{Discusión}

Los resultados del estudio permiten afirmar que los fonoaudiólogos se centran en el escenario salud, dejando en un segundo plano el escenario educación, bienestar social y laboral en los cuales el adulto mayor puede cumplir una función dentro de la sociedad. En el escenario salud, los profesionales de la comunicación humana y sus desórdenes realizan funciones de evaluación diagnóstico e intervención. Lo anterior se encuentra en línea con lo enunciado por Ruiz-Sánchez (2012) quien afirma que el fonoaudiólogo realiza funciones de evaluación e intervención en las dificultades relacionadas con las enfermedades neurodegenerativas. También, confirma lo planteado por Valles (2011), quien propone que el abordaje fonoaudiológico se realiza a través de la estimulación cognitiva-comunicativa para mantener las funciones cognitivas $y$ comunicativas de manera global abarcando todos los aspectos. Finalmente, estos resultados coinciden con la visión de Sánchez, Dumitrache, Calet, \& de los Santos (2016) quien postula que el fonoaudiólogo debe centrar su intervención en los aspectos comunicativos para mantener, activar y/o rehabilitar el lenguaje, el habla, la voz y la deglución, en el caso de las personas que presentan la enfermedad de Alzheimer.

Los resultados también coinciden con los de Montoya \& Rodríguez (2018) quienes reportaron que en Colombia los fonoaudiólogos tienden a desempeñarse en el escenario salud ejerciendo el rol asistencial tal como se da a nivel internacional. En este contexto, el abordaje se centra en la población adulta mayor que se encuentra en clínicas u hospitales y que presentan algún tipo de déficit. De allí que las áreas más trabajadas sean deglución, lenguaje y comunicación. Los resultados del actual estudio concuerdan con los de Rodríguez (2009) quien afirma que el profesional de la comunicación humana y sus desórdenes trabaja con adultos a medida que surge un problema, específicamente en la afasia. Según la autora se hace necesario formular proyectos que permitan describir la comunicación adulta desde un modelo sistémico que contemple la descripción de los procesos y de las habilidades que se evidencian en la dimensión sociocultural, la interpersonal y la individual. De esta manera se podrán generar perfiles comunicativos de los adultos colombianos; y se determinarán patrones de normalidad que respondan a las habilidades reales de la población a partir de las exigencias de los diferentes contextos sociales.

Rodríguez (2009) también plantea que la comunicación en las personas adultas se debe asumir como un conjunto de saberes, habilidades y destrezas que se han construido a lo largo de su ciclo vital y que le permiten comunicarse de manera efectiva con los posibles interlocutores en los diferentes contextos sociales (familia, amigos, trabajo). De allí la importancia de ser analizada desde un modelo en el que se privilegie no sólo lo biológico o lo cognitivo sino como el producto de diversos aprendizajes (social, ideológico, cultural, afectivo, lingüístico, pragmático, entre otros), los cuales se encuentran inscritos dentro de una perspectiva integral del individuo. Es por eso que se hace necesario asumir la comunicación como un proceso intersubjetivo en el que intervienen distintos agentes; como un fenómeno social complejo; como 
un proceso interactivo que permite el intercambio de necesidades, deseos, percepciones, conocimientos o estados emocionales de las personas.

La presente investigación también brinda información sobre el rol investigativo del fonoaudiólogo. En relación a esto, los resultados muestran que, en su gran mayoría los estudios realizados por los encuestados, se enmarcaban en un enfoque médico que se caracteriza por centrar la intervención en la deficiencia. En este contexto, los estudios desarrollados se orientaban a los desórdenes de la comunicación, del lenguaje y de la deglución, los que se centran en la comprensión de las deficiencias que afectan al adulto mayor con envejecimiento patológico. Desde este enfoque se dejan de lado los diferentes contextos donde participan los adultos mayores. Muy pocos estudios aludían a un modelo social teniendo en cuenta todos los contextos, los facilitadores y las barreras que se pueden encontrar en cada uno de ellos. Esto refleja la tendencia de los fonoaudiólogos a trabajar sobre el envejecimiento patológico y no sobre el envejecimiento normal y las percepciones que tienen, tanto el fonoaudiólogo como la sociedad, sobre la adultez mayor y sobre el envejecimiento típico.

Además, trabajar sobre el envejecimiento patológico y no sobre el envejecimiento normal.

Los resultados de esta investigación invitan a los fonoaudiólogos a: 1) reflexionar sobre las acciones que están adelantando con la población adulta mayor para generar calidad de vida y bienestar comunicativo en ellos y en sus familias; 2) difundir los resultados de las investigaciones orientadas a describir el quehacer profesional con el adulto mayor con y sin deficiencias; y 3 ) asumir según Rodríguez \& Pachón (2011) al adulto mayor como un interlocutor que cuenta con un conjunto de saberes relacionados con el proceso comunicativoy con las condiciones necesarias para que éste se dé.

\section{Conclusiones}

El estudio permitió describir las cuatro variables de desempeño de los fonoaudiólogos con la población adulta mayor. En relación con la primera variable, se logró dar una descripción sociodemográfica a nivel nacional de un grupo de fonoaudiólogos colombianos que trabaja con la población adulta mayor, así como del perfil profesional (nivel de estudios, áreas de abordaje y años de experiencia).

Para la variable del quehacer fonoaudiológico, se observó una tendencia alta a ejercer el rol asistencial (66\%). Esto permite sugerir la necesidad de realizar un abordaje más integral y cambiar la visión que los adultos mayores son sujetos sin participación social: La propuesta sería desarrollar estudios que pueden aportar más a los roles docente, investigativo y administrativo en torno a esta población.

Los escenarios en los que más se desempeñan los fonoaudiólogos son Salud con el 70\%, Bienestar Social $23 \%$ y Educación 7\%. Los fonoaudiólogos prestan sus servicios en clínica y en el contexto domiciliario, y dejan en un segundo plano el trabajo los centros de protección social. El enfoque que más se usa en el abordaje es el clínico, observándose una falta de profesionales que busquen dar un enfoque más social, centrado en una intervención en la que se vea al adulto mayor como un ser social y 
participativo.

Las funciones que estos profesionales desarrollan en el rol asistencial, son evaluación-diagnóstico, intervención, asesoría y consejería. Se hace entonces necesario, ir más allá de rehabilitar/habilitar una dificultad. De allí la importancia de plantear propuestas orientadas a potencializar en una población adulta con un envejecimiento típico o sano, su participación social y su bienestar comunicativo.

En el rol administrativo se trabaja en el diseño, ejecución de programas fonoaudiológicos, y en la coordinación de proyectos de mejoramiento social. En el rol investigativo se publican artículos con temáticas centradas en los desórdenes de la deglución, del lenguaje y de la comunicación Además, se observa que muchos de los fonoaudiólogos que trabajan con población adulta mayor no tienen interés por investigar ni documentar sus experiencias para ser difundidas y para ser retroalimentadas por otros profesionales.

Por último, las percepciones que tienen los profesionales acerca de la población adulta mayor, está dada desde la visión de un modelo clínico: De allí que su abordaje se centre en intervenir las deficiencias y/o dificultades que presenta la persona mayor.

\section{Referencias}

Berrío, M. I. (2012). Envejecimiento de la población: un reto para la salud pública. Revista Colombiana de Anestesiología, 40(3), 192-194. https://doi.org/10.1016/j.rca.2012.04.001
Cuervo, C. (1998). La profesión de fonoaudiología: Colombia en perspectiva internacional. Universidad Nacional de Colombia, Santa Fe de Bogotá. Recuperado de http://www.bdigital.unal.edu.co/2344/2/clemenciacuervo echeverri.1998.pdf

Díaz de Rada, V. (2007). Tipos de encuestas considerando la dimensión temporal. Papers. Revista de Sociologia, 86, 131-145. https://doi.org/10.5565/rev/papers/v86n0.814

Dulcey-Ruiz, E., Arrubla, D., \& Sanabria, P. (2013). Envejecimiento y vejez en Colombia (Estudio a Profundidad. Basado en las Encuestas Nacionales de Demografía y Salud - ENDS - 1990 / 2010) (p. 79). Colombia. Recuperado de http://www.profamilia.org.co/docs/estudios/imagenes/3\% 20-

\%20ENVEJECIMIENTO\%20Y\%20VEJEZ\%20EN\%20COLOMBI A.pdf

Escobar, J., \& Cuervo, Á. (2008). Validez de contenido y juicio de expertos: una aproximación a su utilización. Avances en Medición, 6, 27-36.

Grasso, L. (2006). Encuestas. Elementos para su diseño y análisis. Córdoba, Argentina: Editorial Brujas. Recuperado de

https://books.google.cl/books/about/Encuestas_Elemento s_para_su_dise\%C3\%B1o_y_an.html?id=jL_yS1pfbMoC\&re dir_esc=y

Hernández, R., Fernández, C., \& Baptista, M. del P. (2010).

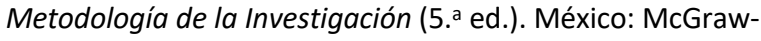
Hill. Recuperado de https://www.esup.edu.pe/descargas/dep_investigacion/M etodologia\%20de\%20la\%20investigaci\%C3\%B3n\%205ta\%2 0Edici\%C3\%B3n.pdf

Millán, J. C. (2006). Principios de geriatría y gerontología. Madrid, España: McGraw-Hill Interamericana de España S.L. Recuperado de https://books.google.cl/books?id=rRTaAAAACAAJ\&dq=\%2 2Principios+de+Geriatr\%C3\%ADa+y+Gerontolog\%C3\%ADa $\% 22 \&$ hl=es-

419\&sa=X\&ved=0ahUKEwjOocfd2YjeAhXDFZAKHabJAHMQ 6AEIKDAA

Montoya, N. C., \& Rodríguez, Y. (2018). Quehacer profesional del fonoaudiólogo con la población adulta mayor: revisión documental. ASALFA, Asociación Argentina de Logopedia, Foniatría y Audiología, 65(1), 33-47.

Petersen, R. C. (2004). Mild cognitive impairment as a diagnostic entity. Journal of Internal Medicine, 256(3), 183194. https://doi.org/10.1111/j.1365-2796.2004.01388.x

Rodríguez, Y. (2009). La competencia comunicativa adulta: producto de un complejo mundo de saberes $y$ experiencias. Areté, 9(1), 6-10. 
Rodríguez, Y., \& Pachón, E. (2011). Estudio descriptivo de la competencia interactiva en adultos sordos señantes. Areté, 11, 102-115.

Ruiz-Sánchez, J. (2012). Estimulación cognitiva en el envejecimiento sano, el deterioro cognitivo leve y las demencias: estrategias de intervención y consideraciones teóricas para la práctica clínica. Revista de Logopedia, Foniatría y Audiología, 32(2), 33-94. https://doi.org/10.1016/j.rlfa.2012.02.002

Sánchez, A., Dumitrache, C., Calet, N., \& de los Santos, M. (2016). Intervención en un paciente con Enfermedad de Alzheimer: anomia léxica. Revista de Investigación en Logopedia, 6(1), 70-87-87.

https://doi.org/10.5209/RLOG.58555

Valles, B. (2011). Representaciones y creencias en torno a la evaluación lingüística del adulto mayor con trastorno cognitivo leve. Revista de Investigación en Logopedia, 1(1), 12-34.

Vargas, L. M. (1994). Sobre el concepto de percepción. Alteridades, 4(8), 47-53. 